\title{
FLORACIÓN Y FRUCTIFICACIÓN DE DIEZ ESPECIES DE PLANTAS DEL CENTRO DE INVESTIGACIÓN Y ENSEÑANZA FORESTAL (CIEFOR), PUERTO ALMENDRA, LORETO, PERÚ
}

\author{
Manuel FLORES ${ }^{1}$, Edward J. ALARCON ${ }^{1}$, Ricardo ZÁRATE ${ }^{2}$, Ana M. RENGIFO ${ }^{3}$, Juan L. FLORES ${ }^{4}$, Juan \\ C. RUIZ ${ }^{5}$ Linder F. MOZOMBITE ${ }^{6}$
}

1 Universidad Nacional de la Amazonía Peruana. Facultad de Ciencias Biológicas. Jr. Pebas cuadra 5, Iquitos, Loreto, Perú. Jr. Pebas cuadra 5, Iquitos, Loreto, Perú. mafloar03@hotmail.com

2. Instituto de Investigaciones de la Amazonía Peruana. Programa de Investigación en Cambio Climático Desarrollo Territorial y Ambiente (Proterra); Av. Quiñones km 2.5, San Juan Bautista, Maynas, Loreto, Perú. rzarate@iiap.org.pe

3. Universidad Nacional de la Amazonía Peruana/Facultad de Ciencias Agronómicas.

4. Universidad Nacional de la Amazonía Peruana/Facultad de Ciencias Forestales.

5. Universidad Nacional de la Amazonía Peruana/Herbarium Amazonense.

6. Servicios de Biodiversidad.

\section{RESUMEN}

Se evaluó mensualmente, durante un periodo de cuatro años (2008-2011), la floración y fructificación de diez especies de angiospermas ( $\mathrm{n}=118$, entre árboles y arbolitos) en la localidad de Puerto Almendra, Loreto, Perú. La floración y fructificación se registró durante la época de alta precipitación, de setiembre a octubre y de noviembre a mayo, respectivamente. Se evaluó el índice de correlación de Pearson entre las variables climáticas (temperatura y precipitación media mensual) y fenológicas (floración y fructificación). Las mayores correlaciones $(0.601$ a 0.629$)$ se dieron entre la temperatura media mensual y la fructificación, con el $40 \%$ de las especies: Iryanthera paraensis Huber (Myristicaceae), Mabea occidentalis Benth. (Euphorbiaceae), Pourouma tomentosa Mart. ex Miq. (Urticaceae) y Protium divaricatum Engl (Burseraceae). Los resultados muestran que la fructificación de la mayoría de las especies del estudio estuvieron influenciadas por la temperatura media mensual, en tanto que la floración estuvo débilmente influenciada por la temperatura; la floración y fructificación de las especies estudiadas no estuvieron notoriamente influenciadas por la precipitación.

PALABRAS CLAVE: Amazonía peruana, CIEFOR, Especies vegetales, Fenología, Floración, Fructificación.

\section{FLOWERING AND FRUITING OF TEN PLANT SPECIES FROM THE RESEARCH AND FORESTRY TEACHING CENTRE (CIEFOR), PUERTO ALMENDRA, LORETO, PERU}

\begin{abstract}
We evaluated flowering and fruiting monthly of ten angiosperm species $(\mathrm{n}=118$, including large and small trees) for a period of four years (2008-2011) in Puerto Almendra, Loreto, Peru. The flowering and fruiting registered during high rainfall period, since september to october and november to may. Pearson's Correlation Index was evaluated between climatic variables (temperature and average annual precipitation) and phenological (flowering and fruiting). The highest correlations (0.601-0.629) were between average monthly temperature and fruiting, with $40 \%$ of species: Iryanthera paraensis Huber (Myristicaceae), Mabea occidentalis Benth. (Euphorbiaceae), Pourouma tomentosa Mart. ex Miq. (Urticaceae) y Protium divaricatum Engl (Burseraceae). According to the results, the fruiting of most species were influenced by the average monthly temperature, by contrast, the flowering of species were weakly influenciated. The flowering and fruiting of species studied were not influenced considerably by precipitation. Finally, we are showing the characteristics of the flowers and fruits from the species.
\end{abstract}

KEYWORDS: CIEFOR, Flowering, Fruiting, Peruvian amazon, Phenology, Plant species. 


\section{INTRODUCCIÓN}

Conceptualizamos la floración y fructificación como la temporada en la que se producen las flores o frutos, indicando los meses y las características principales del clima (Font, 1985). La floración y fructificación de plantas neotropicales están influenciadas por factores abióticos y bióticos (Pérez \& Martinez-Laborde, 1994). Entre los factores abióticos encontramos al clima (Castro, 1987; Tello, 1996; Villasana \& Suárez, 1997) y al suelo principalmente. Dentro de los factores bióticos encontramos tanto los genéticos (Rathcke \& Lacey, 1985; Alencar, 1994); fisiológicos (Alencar, 1994), como las interrelaciones planta-animal (polinizadores, dispersores y depredadores) y planta-planta (Foster, 1990; Bullock et al., 1983, Rathcke \& Lacey, 1985; Castro, 1987; Alencar, 1994; Villasana \& Suárez, 1997). Esta información ayuda a comprender mejor la dinámica de los bosques y favorece el adecuado manejo de las plantas.

La literatura científica reporta para la Amazonía peruana un número todavía muy deficiente de data en lo que respecta a los procesos de floración y fructificación de las plantas (Gautier \& Spichiger, 1986; Baluarte, 1996; Freitas \& Peter, 2000; Ruiz, 2000; Torres, 2001; Zárate et al., 2006). Estas investigaciones incluyen aproximadamente 209 especies que representan solamente el $3.4 \%$ de angiospermas y gimnospermas del total de 6.237 (Vásquez \& Rojas, 2006). Adicionalmente, a partir de los datos recogidos en cuatro herbarios peruanos por Flores (2008), también se reporta la floración y fructificación de 2.308 especies de angiospermas, soteniendo el autor que la floración en la Amazonía peruana acontece aproximadamente en los meses de menor precipitación, extendiéndose hasta los meses lluviosos, concordando con otras investigaciones (Gautier \& Spichiger (1986), Baluarte (1996), Ruiz (2000) y Zárate et al. (2006)). Es necesario ampliar los conocimientos sobre la floración y frutificación de otras especies arbóreas como: Alchornea triplinervia (Spreng.) Müll.Arg., Cedrelinga cateniformis (Ducke) Ducke, Eschweilera coriacea (DC.) S.A.Mori, Iryanthera paraensis Huber, Leonia cymosa Mart., Mabea occidentalis Benth., Pourouma tomentosa Mart. ex Miq., Protium divaricatum Engl., Rinorea lindeniana (Tul.) Kuntze, Zygia basijuga (Ducke) Barneby \& J.W. Grimes, entre otras, cuya importancia radica en su uso directo e indirecto, tanto en la construcción de muebles e inmuebles, en la alimentación, en la medicina, como para el beneficio de fibras para ataduras y otras aplicaciones (Vásquez, 1997; Rutter, 1990; Vásquez, 1989).
El presente trabajo pretende contribuir a ocupar los vacios de información sobre estas especies, a través de tres objetivos específicos: a) monitoreo de la floración y frutificación durante cuatro años, b) medir la influencia de la temperatura y la precipitación en estos períodos fenológicos y c) reportar las características morfológicas de flores y frutos.

\section{MATERIAL Y MÉTODOS}

\section{Área de estudio}

El presente trabajo se llevó a cabo en el Centro de Investigación y Enseñanza Forestal (CIEFOR), ubicado en la inmediaciones del centro poblado Puerto Almendra, en el distrito de San Juan Bautista, provincia de Maynas, departamento de Loreto, Amazonía peruana. Las parcelas estan ubicadas en las coordenadas que se detallan en la Tabla 1 y Figura 1. La cubierta vegetal está formada por árboles, arbustos, lianas, etc. Los árboles son de porte mediano o, excepcionalmente, de gran porte. Por las características geomorfológicas el área está catalogada como terraza media a alta. El clima es variado, la temperatura varía entre $22^{\circ} \mathrm{C}$ y $34^{\circ} \mathrm{C}$ (Figura 2) y la precipitación anual bordea los 2.400 mm (Figura 3). La vegetación corresponde a bosques no inundables. El suelo presenta textura arcillosa (parcelas 1, 2, 3, 4, 5 y 6) y arenosa (parcelas 7, 8, 9 y 10).

Se delimitaron diez parcelas de $20 \times 50 \mathrm{~m}$, ubicadas cada $100 \mathrm{~m}$ la una de la otra, a lo largo de una trocha principal en dirección norte - sur. Cada parcela fue subdividida en subparcelas de $10 \mathrm{~m}$ x 10 $\mathrm{m}$. Luego se procedió a codificar las plantas estudiadas y se midió la altura total y el DAP (diámetro a la altura del pecho (DAP)). Solamente estudiamos los individuos que superaban los $3 \mathrm{~cm}$ de DAP.

\section{Selección de especies e individuos}

Las especies estudiadas fueron: Alchornea triplinervia (Spreng.) Müll.Arg (Euphorbiaceae), Cedrelinga cateniformis (Ducke) Ducke, (Fabaceae), Eschweilera coriacea (DC.) S.A.Mori (Lecythidaceae), Iryanthera paraensis Huber (Myristicaceae), Leonia cymosa Mart. (Violaceae), Mabea occidentalis Benth. (Euphorbiaceae), Zygia basijuga (Ducke) Barneby \& J.W.Grimes (Fabaceae), Pourouma tomentosa Mart. ex Miq. (Urticaceae), Protium divaricatum Engl. (Burseraceae) y Rinorea lindeniana (Tul.) Kuntze (Violaceae). Estas especies se seleccionaron por su importancia ecológica (dominancia por cantidad de individuos) y socioeconómica. 


\section{MAPA DE UBICACIÓN DE LAS PARCELAS}

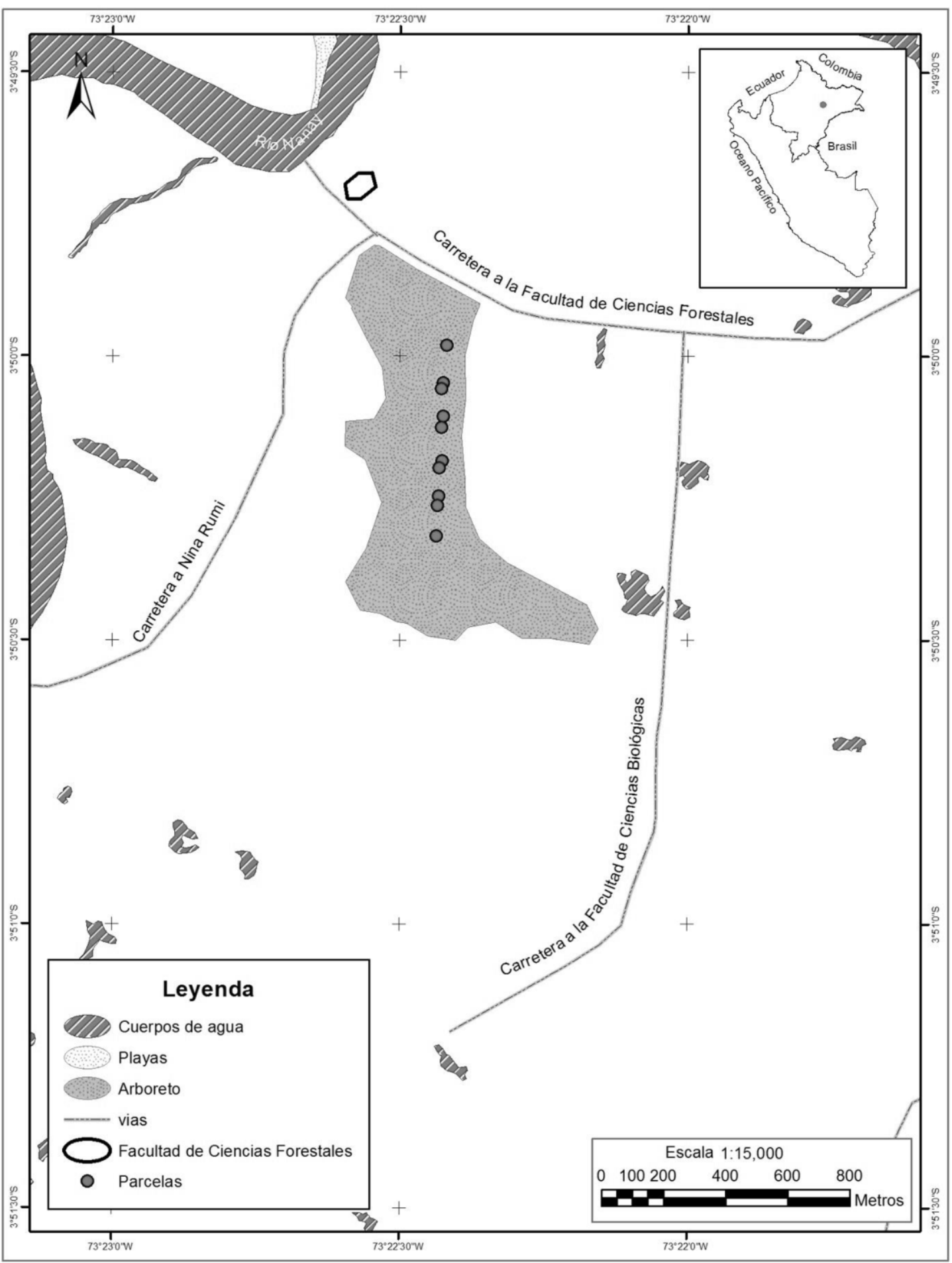

Figura 1. Mapa de ubicación del área de estudio, CIEFOR, Puerto Almendra, UNAP (Loreto, Perú). 
Los individuos se seleccionaron en base a: 1.Edad adulta. 2.- No presentar cortes o rajaduras y estar completamente erguidos. Y 3.- Abundancia disponible de individuos adultos (al menos 7 individuos).

Para la información de las características dasométricas, ver la Tabla 2.

\section{Registro de la floración, fructificación, precipitación y temperatura}

Las observaciones de la floración y fructificación fueron hechas mensualmente con ayuda de binoculares. Las observaciones fueron realizadas durante cuatro años, desde enero del 2008 hasta diciembre del 2011 (48 meses). Las fenofases consideradas son: flores, fruto inmaduro, fruto maduro y fruto postmaduro.

Los datos de precipitación y temperatura fueron obtenidos del Servicio Nacional de Meteorología e Hidrología del Perú (SENAMHI), Iquitos, Estación Meteorológica de San Roque, ubicada a $12 \mathrm{~km}$ aproximadamente de las parcelas establecidas.

\section{Identificación de las especies y caracterización de las flores y frutos}

La identificación de las especies se realizó en el Herbario Amazonense (AMAZ). Las colecciones botánicas de estas especies están depositadas en el Herbario Amazonense (AMAZ) y Herbario San Marcos (USM) y corresponden a las colecciones realizadas por Manuel Flores (códigos MF2500MF2536). Las flores $y / 0$ frutos colectados o recolectados fueron fotografiados y colocados en bolsitas de plástico; se estudió su morfología en las instalaciones del AMAZ. Las muestras de flores y frutos fueron preservadas en alcohol al $70 \% \mathrm{y}$ depositadas en el Herbario Amazonense (AMAZ), a fin de incrementar el acervo de su carpoteca.

\section{Análisis de los datos}

Los datos de floración y fructificación fueron correlacionados con los de precipitación y temperatura de la estación meteorológica de San Roque. Se analizó con el índice de correlación de Pearson.

Tabla 1. Coordenadas geográficas de las diez parcelas establecidas en el CIEFOR, Puerto Almendra, UNAP (Loreto, Perú).

\begin{tabular}{cccc}
\hline Parcela & Longitud & Latitud & Altitud (msnm) \\
\hline Parcela 1 & $73^{\circ} 22^{\prime} 25.37^{\prime \prime} \mathrm{W}$ & $3^{\circ} 49^{\prime} 58.79^{\prime \prime} \mathrm{S}$ & 114 \\
\hline Parcela 2 & $73^{\circ} 22^{\prime} 25.69^{\prime \prime} \mathrm{W}$ & $3^{\circ} 50^{\prime} 2.76^{\prime \prime} \mathrm{S}$ & 107 \\
\hline Parcela 3 & $7^{\circ} 22^{\prime} 25.88^{\prime \prime} \mathrm{W}$ & $3^{\circ} 50^{\prime} 3.38^{\prime \prime} \mathrm{S}$ & 113 \\
\hline Parcela 4 & $73^{\circ} 22^{\prime} 25.71^{\prime \prime} \mathrm{W}$ & $3^{\circ} 50^{\prime} 6.31^{\prime \prime} \mathrm{S}$ & 106 \\
\hline Parcela 5 & $73^{\circ} 22^{\prime} 25.91^{\prime \prime} \mathrm{W}$ & $3^{\circ} 50^{\prime} 7.48^{\prime \prime} \mathrm{S}$ & 101 \\
\hline Parcela 6 & $73^{\circ} 22^{\prime} 25.80^{\prime \prime} \mathrm{W}$ & $3^{\circ} 50^{\prime} 11.03^{\prime \prime} \mathrm{S}$ & 105 \\
\hline Parcela 7 & $73^{\circ} 22^{\prime} 26.12^{\prime \prime} \mathrm{W}$ & $3^{\circ} 50^{\prime} 11.75^{\prime \prime} \mathrm{S}$ & 107 \\
\hline Parcela 8 & $73^{\circ} 22^{\prime} 26.18^{\prime \prime} \mathrm{W}$ & $3^{\circ} 50^{\prime} 14.74^{\prime \prime} \mathrm{S}$ & 110 \\
\hline Parcela 9 & $73^{\circ} 22^{\prime} 26.28^{\prime \prime} \mathrm{W}$ & $3^{\circ} 50^{\prime} 15.72^{\prime \prime} \mathrm{S}$ & 113 \\
\hline Parcela 10 & $73^{\circ} 22^{\prime} 26.40^{\prime \prime} \mathrm{W}$ & $3^{\circ} 50^{\prime} 18.94^{\prime \prime} \mathrm{S}$ & 112 \\
\hline
\end{tabular}




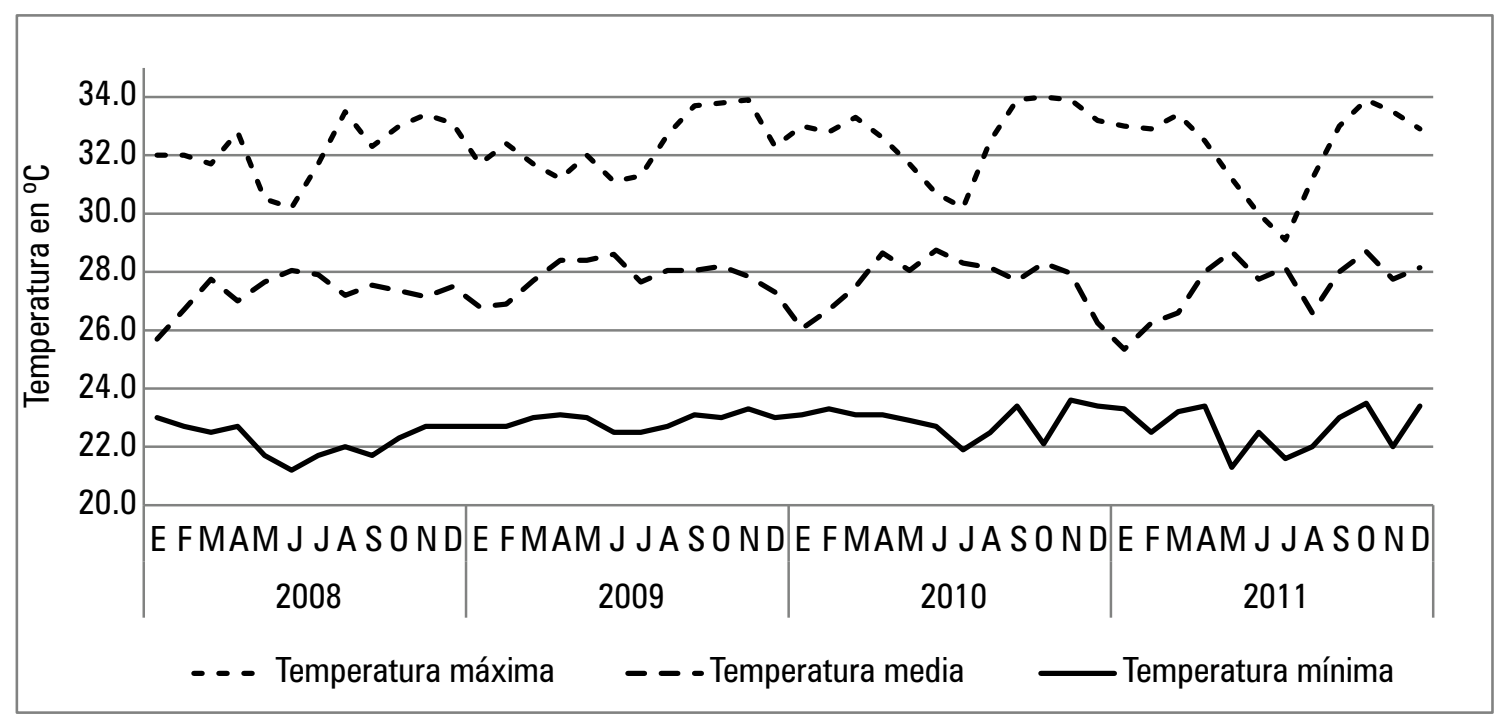

Figura 2. Temperatura en ${ }^{\circ} \mathrm{C}$ de la estación meteorológica de San Roque (Iquitos) de 4 años.

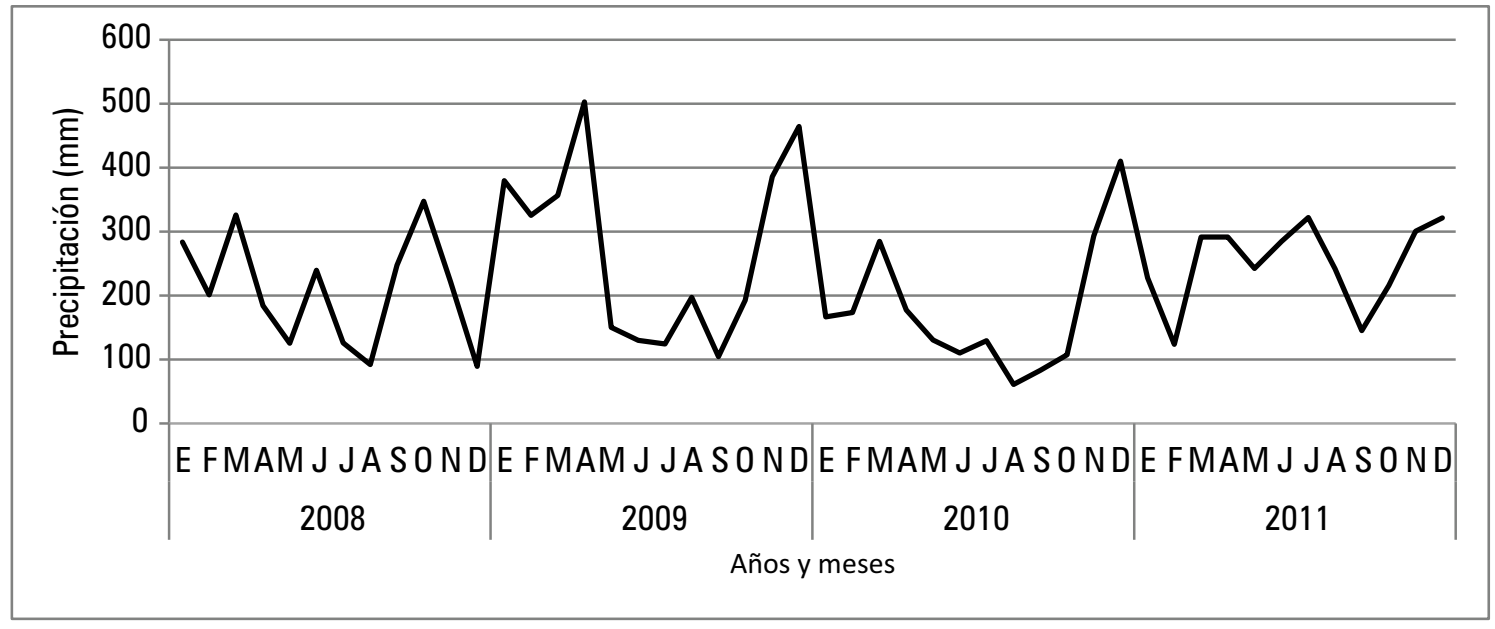

Figura 3. Precipitación (mm) de la estación meteorológica de San Roque (Iquitos) durante cuatro años. 
Tabla 2. Características de las plantas muestreadas en el CIEFOR, Puerto Almendra, UNAP (Loreto, Perú).

\begin{tabular}{rlccccc}
\hline \multirow{2}{*}{ ID } & \multirow{2}{*}{ Especie } & \multirow{2}{*}{$\begin{array}{c}\mathbf{N}^{\mathbf{0}} \text { de ind. } \\
\text { estudiados }\end{array}$} & \multicolumn{2}{c}{ DAP cm } & \multicolumn{2}{c}{ Altura } \\
\cline { 5 - 7 } & & 7 & $10.3-25$ & 16.1 & $16-20$ & 15.3 \\
\hline 1 & Alchornea triplinervia (Spreng.) Müll.Arg. & 7 & $12-150$ & 81.0 & $12-30$ & 18.2 \\
2 & Cedrelinga cateniformis (Ducke) Ducke & 22 & $50-180$ & 95.0 & $15-25$ & 19.3 \\
3 & Eschweilera coriacea (DC.) S.A. Mori & 8 & $16.7-50$ & 23.5 & $10-20$ & 15.2 \\
\hline 4 & Iryanthera paraensis Huber & 7 & $3.5-12$ & 6.4 & $5-10$ & 8.5 \\
5 & Leonia cymosa Mart. & 13 & $5-15$ & 10.5 & $5-16$ & 8.7 \\
6 & Mabea occidentalis Benth. & 11 & $20-40$ & 32.0 & $10-18$ & 14.0 \\
7 & Pourouma tomentosa Mart. ex Miq. & 7 & $7.3-30$ & 16.3 & $8-20$ & 12.5 \\
8 & Protium divaricatum Engl. & 26 & $5-12$ & 10.5 & $7-12$ & 8.8 \\
9 & Rinorea lindeniana (Tul.) Kuntze & 10 & $10-18$ & 15.0 & $9-15$ & 12.0 \\
\hline 10 & Zygia basijuga (Ducke) Barneby \& J.W. Grimes & & & & & \\
\hline
\end{tabular}

\section{RESULTADOS}

\section{Floración y fructificación}

Los resultados muestran un patrón regular de floración y fructificación. La floración coincide generalmente con el período de inicio de las lluvias (setiembre a octubre) y la fructificación con el periodo de mayor intensidad de las lluvias (noviembre a mayo). La especie Leonia cymosa tiende a fructificar casi todo el año, al menos durante el 2009. Mientras que las especies Zygia basijuga, Protium divaricatum y Pourouma tomentosa, tienden a presentar un solo pico de fructificación hacia los meses de setiembre-diciembre. Por su parte, las especies Alchornea triplinervia, Mabea occidentalis, Cedrelinga cateniformis y Eschweilera coriacea fructifican principalmente de setiembre a marzo (Tabla 4).

A continuación se presenta los datos de floración y fructificación de cada una de las diez especies investigadas:

\section{Alchornea triplinervia (Spreng.) Müll.Arg.}

La floración ocurre en el mes de agosto y las fenofases de fruto inmaduro, fruto maduro y fruto postmaduro ocurren en setiembre-octubre, pero también se pudo observar en los primeros meses del año.
2. Cedrelinga cateniformis (Ducke) Ducke.

Los períodos de floración se presentan en los meses de setiembre a octubre, los primeros botones se observan a partir de la tercera semana de setiembre. La fructificación se produce entre los meses de noviembre a febrero. Los frutos se encuentran en la fenofase fruto inmaduro en noviembre-diciembre, fruto maduro en enerofebrero y fruto postmaduro a finales de febrero.

\section{Eschweilera coriacea (DC.) S.A.Mori}

La floración se produce entre los meses de octubre y noviembre. La fructificación entre los meses de noviembre a marzo, pudiéndose extender hasta abril. La etapa y tiempo de desarrollo de la fenofase fruto inmaduro ocurre de noviembre a enero, fruto maduro de febrero a marzo y fruto postmaduro (desprendimiento del opérculo) de marzo a abril.

\section{Iryanthera paraensis Huber}

El periodo aproximado de floración es de tres a cuatro meses, de julio a octubre. El período aproximado de fructificación es de cinco meses, de noviembre a marzo. La etapa y tiempo de desarrollo de la fenofase fruto inmaduro se desarrolla de setiembre a diciembre, fruto maduro de enero a febrero y fruto postmaduro (dehiscentes, incluso en el suelo) de febrero a marzo. 


\section{Leonia cymosa Mart.}

La floración se produce a partir de la tercera semana de septiembre y llega hasta octubre. La fructificación ocurre a partir de noviembre hasta junio o julio. La fenofase fruto inmaduro ocurre de noviembre a febrero, fruto maduro de marzo a junio y fruto postmaduro (caídos) en julio.

\section{Mabea occidentalis Benth.}

La floración se produce entre agosto y setiembre. La fructificación entre setiembre y febrero. La etapa y tiempo de desarrollo de la fenofase fruto inmaduro ocurre de setiembre a octubre, fruto maduro de noviembre a diciembre y postmaduro de enero a febrero.

\section{Pourouma tomentosa Mart. ex Miq.}

La floración se produce entre setiembre a octubre. La fructificación se observa entre octubre a febrero, en ocasiones hasta marzo. La etapa y tiempo de desarrollo de la fenofase fruto inmaduro ocurre de octubre a noviembre, fruto maduro de noviembre a diciembre y fruto postmaduro de enero-febrero (en algunos casos hasta marzo).

\section{Protium divaricatum Engl.}

La floración se produce entre agosto-octubre, mientras que la fructificación se observa entre octubre a marzo. La etapa y tiempo de desarrollo de la fenofase fruto inmaduro ocurre de octubre a diciembre, fruto maduro de diciembre a febrero y fruto postmaduro de febrero a marzo.

\section{Rinorea lindeniana (Tul.) Kuntze}

La floración se produce entre setiembre a noviembre y la fructificación entre noviembre a enero. La etapa y tiempo de desarrollo de la fenofase fruto inmaduro ocurre en noviembre, fruto maduro en diciembre y fruto postmaduro en enero.

\section{Zygia basijuga (Ducke) Barneby \& J.W. Grimes}

$\mathrm{Su}$ periodo de floración empieza en agosto y termina en octubre. La fructificación es de 4 a 5 meses, de noviembre a marzo. La etapa y tiempo de desarrollo de la fenofase de fruto inmaduro ocurre de octubre a noviembre, fruto maduro de diciembre a febrero y fruto postmaduro de marzo a mayo, esta fase puede prolongarse hasta la siguiente floración.

Tabla 3. Correlaciones de la temperatura $\left(T^{\circ}\right)$ y la precipitación (Pp) con la floración (fl) y fructificación (fr) de 10 especies del CIEFOR Puerto Almendra, UNAP, Loreto.

\begin{tabular}{|c|c|c|c|c|c|c|}
\hline ID & Especie & $\begin{array}{l}N^{0} \text { de ind. } \\
\text { estudiados }\end{array}$ & $T^{0}$ vs fl & $T^{0}$ vs fr & Pp vs fl & Pp vs fr \\
\hline & Alchornea triplinervia (Spreng.) Müll.Arg. & 7 & 0.1959 & -0.095 & -0.357 & 0.2252 \\
\hline 1 & Cedrelinga cateniformis (Ducke) Ducke & 7 & 0.3915 & 0.3878 & 0.3696 & -0.002 \\
\hline 2 & Eschweilera coriacea (DC.) S.A. Mori & 22 & 0.3009 & 0.2209 & $0.5134-$ & 0.2378 \\
\hline 3 & Iryanthera paraensis Huber & 8 & 0.0498 & 0.6027 & 0.048 & 0.277 \\
\hline 4 & Leonia cymosa Mart. & 7 & 0.3939 & 0.223 & 0.5178 & -0.044 \\
\hline 5 & Mabea occidentalis Benth. & 13 & -0.091 & 20.6014 & 0.1464 & 0.2672 \\
\hline 6 & Zygia basijuga (Ducke) Barneby \& J.W. Grimes & 10 & 0.114 & 0.5226 & 0.4812 & 0.0862 \\
\hline 7 & Pourouma tomentosa Mart. ex Miq. & 11 & 0.0718 & 0.6092 & 0.4853 & 0.1824 \\
\hline 8 & Protium divaricatum Engl. & 7 & 0.0718 & 0.6288 & 0.4854 & 0.232 \\
\hline 9 & Rinorea lindeniana (Tul.) Kuntze & 26 & 0.4604 & 0.3199 & 0.2698 & -0.113 \\
\hline 10 & Total & 118 & & & & \\
\hline
\end{tabular}




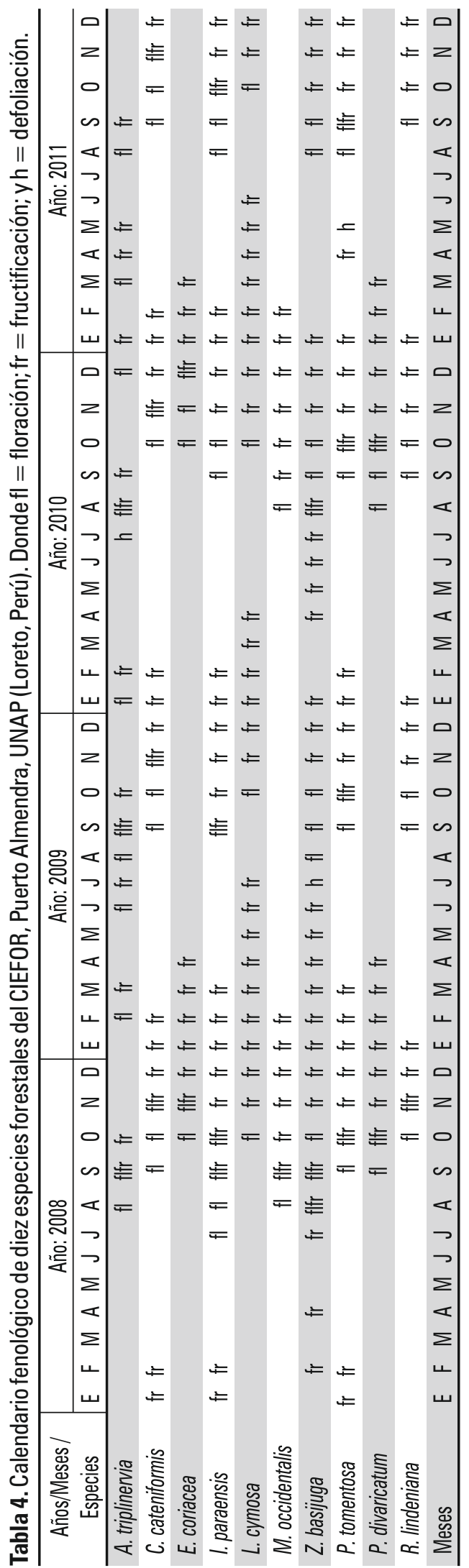




\section{Valores de temperatura y precipitación}

El rango de variación de la temperatura máxima para la estación de San Roque (Loreto) fue de $29.1^{\circ} \mathrm{C}$ a $34^{\circ} \mathrm{C}$, la temperatura mínima varió de $21.3^{\circ} \mathrm{C}$ a $23.6^{\circ} \mathrm{C}$ y la temperatura media varió de $25.4^{\circ} \mathrm{C}$ a $29^{\circ} \mathrm{C}$ durante los años de observación de la floración y fructificación en el CIEFOR, comprendidos entre el 2008 al 2011. Del mismo modo, los datos de precipitación de la estación de San Roque variaron de $61.1 \mathrm{~mm}$ en agosto del 2010 a $464.5 \mathrm{~mm}$ en diciembre del 2009.

\section{Índice de correlación de Pearson entre las fenofases y el clima}

La correlación de Pearson entre la floración de las diez especies y la temperatura media mensual varió de -0.091 en Mabea occidentalis a 0.4604 en Rinorea lindeniana, con un promedio de 0.1959 . La correlación es positiva para todas las especies, excepto para Mabea occidentalis (Tabla 3). Usando solo valores absolutos de correlación de la floración de las diez especies y la temperatura, tenemos que la desviación estándar es de 0.1581 , con un coeficiente de variación de $73.85 \%$. La correlación entre la fructificación de las diez especies y la temperatura media mensual varió de -0.095 en Alchornea triplinervia, a 0.6288 en Protium divaricatum, con un promedio de 0.4022 . La correlación es positiva para todas las especies excepto para Alchornea triplinervia. Teniendo en cuenta solo los valores absolutos de esta correlación, se tiene una desviación estándar de 0.1976 , con un coeficiente de variación de $46.91 \%$.

La correlación entre la floración de las diez especies y la precipitación total mensual varió de 0.048 en Iryanthera paraensis a 0.5178 en Leonia cymosa, con un promedio de 0.2934 . La correlación es positiva para todas las especies, excepto para Alchornea triplinervia e Iryanthera paraensis. Usando solo valores absolutos de la correlación de la floración de las diez especies y la precipitación total mensual, tenemos que la desviación estándar es de 0.1651 con un coeficiente de variación de $44.93 \%$. La correlación entre la fructificación de las diez especies y la precipitación total mensual varió de 0.002 en Cedrelinga cateniformis a 0.277 en Iryanthera paraensis, con un promedio de 0.1349 . La correlación es positiva para siete especies y negativa para Cedrelinga cateniformis, Leonia cymosa y Rinorea lindeniana. Usando solo valores absolutos de la correlación de la fructificación de las diez especies y la precipitación total mensual, tenemos que la desviación estándar es de: 0.0982, con un coeficiente de variación de $58.92 \%$ (tabla 3 ).

\section{Características botánicas con énfasis en flores y frutos}

\section{Alchornea triplinervia (Spreng.) Muell. Arg.}

Nombre local: "zancudo caspi"

Las inflorescencias son espigas reunidas en grupos al final de las ramitas; flores unisexuales, apétalas, flores masculinas con cáliz 2 a 5 partido, ocho estambres con filamentos soldados en un disco pequeño; flor femenina terminal, 3-6 sépalos, 2-3 ovario locular, estilos libres o basalmente connados, estigmas persistentes; fruto cápsula compuesto de 23 cocos o subglobosa, lisa, tuberculada, semillas sin carúncula. Plantas dioica, raramente monoica.

\section{Cedrelinga cateniformis $L$.}

Nombre local: "tornillo"

Inflorescencia en cabezuelas, organizadas en panículas (López, 1981 ), de 10 a $25 \mathrm{~cm}$ de largo, agrupadas en manojos, las cabezuelas de 2 a $3 \mathrm{~cm}$ de largo, con pedúnculo de $1 \mathrm{a} 2 \mathrm{~cm}$ de largo; flores pequeñas hermafroditas de 1 a $1.5 \mathrm{~cm}$ de largo, actinomorfas con cáliz y corola presentes, el cáliz pequeño de 1 a $2 \mathrm{~mm}$ de largo, tubular con 5 dientes; androceo con numerosos estambres de 1 a $1.5 \mathrm{~mm}$ de largo, el pistilo único con un estilo largo y estigma obsoleto; los botones florales miden menos de $1 \mathrm{~mm}$ de diámetro. Frutos legumbres muy largos y aplanados de 20 a $35 \mathrm{~cm}$ de largo y 2 a $3 \mathrm{~cm}$ de ancho, con 6 a 15 semillas, la legumbre estrechada entre las semillas y revirada helicoidalmente. Plantas hermafroditas.

\section{Eschweilera coriacea (A. DC) S. Mori}

Nombre local: "machimango colorado"

Inflorescencia en racimos terminales y axilares, a menudo cortamente subdividas (panícula) de unos 8 a $15 \mathrm{~cm}$ de largo y 5 a $8 \mathrm{~cm}$ de ancho con pocas flores; flores grandes de 2.5 a $3.5 \mathrm{~cm}$ de largo y de 2 a $3 \mathrm{~cm}$ de diámetro, hermafroditas, zigomorfas con cáliz y corola presentes, el cáliz con 6 sépalos anchamente ovados de 7 a $9 \mathrm{~mm}$ de largo, la corola con 6 pétalos de color amarillo blanquecino, el androceo fuertemente asimétrico con numerosos estambres ubicados en la base de una estructura en forma de caperuza, esta doblemente enroscada hacia adentro, color amarillo intenso, el gineceo con ovario ínfero con 2 lóculos o cavidades. Fruto en forma de una pequeña olla (pixidio), anchamente cónica de unos 3 a $5 \mathrm{~cm}$ de largo y de 2.5 a $4 \mathrm{~cm}$ de diámetro, muy leñosas, el opérculo o tapa de $2 \mathrm{a} 3 \mathrm{~cm}$ de altura, en interior de este con pocas semillas, ellas suborbiculares no aladas, ariladas carnosas. Plantas hermafroditas. 


\section{Iryanthera paraensis Huber}

Nombre local: "cumalilla colorada"

Las inflorescencias estaminadas, generalmente flexuosas, de 5 a $20 \mathrm{~cm}$ de largo, flores de 7 a 20 por fascículos, pedicelo más de $3 \mathrm{~mm}$ de largo, perianto cupuliforme o subrotaceo de 0.5 a $1.5 \mathrm{~mm}$ de largo, partido casi hasta la base, androceo de 0.5 a $1 \mathrm{~mm}$ de largo, anteras unidas dorsalmente de 0.2 a $0.3 \mathrm{~mm}$ de largo; inflorescencias pistiladas de 1 a $5 \mathrm{~cm}$ de largo, rameales (caulógenas en el ápice), flores de 10 a 20 por fascículos, pedicelos más de $4 \mathrm{~mm}$ de largo, pistilo subcilíndrico, fruto cápsulas subcarinadas de 8 a 22 x 24 a 28 mm, de acuerdo a Vásquez (1997) con algunas modificaciones. Plantas dióicas.

\section{Leonia cymosa Mart.}

Nombre local: "aceituna caspi"

Inflorescencias axilares o rameales en cimas definidas, flores con sépalos de 1 a $1.5 \mathrm{~mm}$ de largo, pétalos de 3 a $4 \mathrm{~mm}$ de largo, anaranjados o amarillentos, frutos drupas de 2 a $3 \mathrm{~cm}$ de diámetro, amarillos y dulces al madurar. Plantas hermafroditas.

\section{Mabea occidentalis Benth.}

Nombre local: "shiringuilla"

Presenta inflorescencias axilares o subterminales, 1 (2), pedúnculos de 14 a $18 \mathrm{~cm}$ de largo; las flores estaminadas en panículas espiciformis de $12 \times 25 \mathrm{~cm}$, brácteas con apariencia sésil, glándulas lisas; frutos cápsulas de $15 \mathrm{~mm}$ de largo de color marrón, densamente tomentoso (Vásquez, 1997). Plantas monoicas.

\section{Pourouma tomentosa Mart.}

Nombre local: "sacha uvilla"

Las inflorescencias masculinas miden 12 × 12 $\mathrm{cm}$, pedúnculo de 2 a $5 \mathrm{~cm}$ de largo, éste y las ramificaciones densamente pubérulos, con tricomas aracnoides. Flores estaminadas sésiles o pediceladas en cabezuelas subglobosas de 3 a $4 \mathrm{~mm}$ de diámetro con tépalos unidos; inflorescencias femeninas en fruto de $20 \times 13 \mathrm{~cm}$, pedúnculo de 2 a $8 \mathrm{~cm}$ de largo, flores pistiladas, pediceladas o subsésiles, usualmente en 2 grupos; fruto drupas oblongoides o subglobosas a ovoides de 15 a 20 × 8 a $15 \mathrm{~mm}$, negras o purpuras, generalmente amarillos pubérulas a velutinas o subhirtulas (Vásquez, 1997). Plantas generalmente dioicas.

\section{Protium divaricatum Engl.}

Nombre local: "copal blanco"
Las inflorescencias son panículas axilares o subterminales de 10 a $15 \mathrm{~cm}$ de largo, flores pequeñas con cáliz y corola presente, pentámeras, unisexuales por reducción de uno de los sexos, el pedicelo de 1 a $2 \mathrm{~mm}$ de largo, cáliz $1 \mathrm{~mm}$ de largo, 5 dentados, los pétalos 5 libres blanquecinos, los estambres 10 de cerca de $1 \mathrm{~mm}$ de largo, el gineceo con un pistilo diminuto, el ovario súpero, el estilo corto y el estigma capitado. Frutos drupas subelipsoides glabros de $2 \times 3 \mathrm{~cm}$, pericarpio herbáceo. Plantas dioicas.

\section{Rinorea lindeniana (Tul.) Kuntze}

Nombre local: "trompetero caspi" "cafecillo"

Las inflorescencias son racimos de 7 a $15 \mathrm{~cm}$, las flores presentan sépalos de $1 \mathrm{~mm}$ de largo, pubérulos por fuera, densamente ciliados, pétalos $3.5 \mathrm{~mm}$ de largo, estambres y apéndice de $2 \mathrm{~mm}$ de largo, anteras con apéndice dentado-lacerados, pistilo hirsuto, Frutos cápsulares de $25 \mathrm{~mm}$ de largo, glabros (Vásquez, 1997). Plantas hermafroditas.

\section{Zygia basijuga (Ducke) Barneby \& J.W.Grimes}

Nombre local: "bushilla" "pashaquilla"

Inflorescencias en racimos de glomérulos, de 3 a $5 \mathrm{~cm}$ de largo; flores con cáliz de 1 a $1.5 \mathrm{~mm}$ de largo, subglabros, corola de 8 a $12 \mathrm{~mm}$ de largo; sus frutos son legumbres, rectos a curvados de 13 a $20 \times 1.5 \mathrm{~cm}$. Plantas hermafroditas.

\section{DISCUSIÓN}

\section{Floración}

La floración en siete de las diez especies evaluadas ocurrió de setiembre a octubre, mientras que en las tres especies restantes (Iryanthera paraensis, Mabea occidentalis y Zygia basijuga) se dio antes de setiembre, coincidiendo generalmente con el período de inicio de las lluvias, concordando con Zárate et al. (2006) para especies de los bosques sobre arena blanca y arcilla de la Reserva Nacional Allpahuayo Mishana (Amazonía peruana). Estos resultados coiciden parcialmente con los encontados por Flores (2008) para 2.308 especies de angiospermas en la Amazonía peruana, quien a través del análisis de información de exicatas de herbarios reportó un periodo de floración un poco más amplio (julio a diciembre, con un mayor pico en agosto). Al igual que Alencar (1994) que reportó para cinco especies de Sapotaceae en la Amazonía brasilera (Reserva Ducke) el mayor periodo de floración de julio a noviembre. En este caso, coincide con el periodo de menor precipitación. El mayor periodo de floración fue reportado por 
Henderson et al. (2000) para 27 especies de palmeras en la Amazonía brasilera, señalando que estas especies florecen durante todo el año. Un análisis de los reportes de 1.249 especímenes de las diez especies evaluadas, a partir de datos de herbario del Missouri Botanical Garden de 2014 (www.tropicos), nos permite señalar que la floración se produce de julio a diciembre para toda la cuenca amazónica con diferentes periodos e intensidades de precipitación. Entonces, el momento de la floración puede tender a coincidir en los últimos seis meses del año y ocurrir bajo diferentes intensidades de precipitación pluvial para diferentes lugares.

\section{Fructificación}

La fructificación de varias especies coincide con el periodo de mayor intensidad de las lluvias (noviembre a mayo), coincidiendo con lo publicado por Zárate et al. (2006), Ruiz (2000), Baluarte (1995) y Alencar (1994). Un análisis más profundo del Missouri Botanical Garden, en base a los reportes de 1.249 especimenes de las diez especies evaluadas en la Cuenca amazónica, muestra que la frutificación ocurre principalmente de setiembre a marzo.

\section{Floración y fructificación por especie}

Los periodos de floración y frutificación, para algunas de las especies evaluadas en este estudio, fueron relativamente semejantes con los reportados por otros estudios como el caso de Zárate et al. (2006) y Flores (2008), quienes reportaron que Alchornea triplinervia fructifica en el mes de octubre e I. paraensis florece en noviembre y fructifica en diciembre en plena época de lluvias; mientras que en el presente estudio la floración de $I$. paraensis fue de julio a septiembre y Alchornea triplinervia puede tener períodos irregulares en dichos estados fenológicos.

Algunas especies como Cedrelinga cateniformis, Leonia cymosa, Pourouma tomentosa y Rinorea lindeniana exhiben un patrón de floración y fructificación regular y son monomodales; de acuerdo a Reynel et al. (2003), la floración de $C$. cateniformis mayormente se realiza a fines de la estación menos lluviosa (entre noviembre y diciembre) y la fructificación a inicios de la estación de lluvia (entre diciembre y febrero).

Aróstegui \& Díaz (1992), sugieren que el proceso desde la floración hasta la maduración del fruto demora cinco meses para algunas especies forestales. En esta investigación presentamos resultados similares, se observó la floración en los meses de setiembre a octubre y la fructificación se produce entre los meses de noviembre a febrero; y entre ambas fenofases hay un tiempo de 6 meses.

En el caso de Leonia cymosa (Pfrommer \& Würzburg, 2009), para la Amazonía ecuatoriana (Estación Biológica de Cuyabeno), florece dos veces al año, uno entre los meses de febrero a marzo y otro en el mes de octubre. Estos autores afirman también que los eventos de fructificación se produjeron, uno, entre marzo y mayo $\mathrm{y}$ otro, entre agosto $\mathrm{y}$ septiembre. Mientras que en la presente investigación la fructificación fue de noviembre hasta junio. Esta especie puede tener de uno a dos periodos de floración y fructificación durante el año en diferentes lugares de la Amazonía. Asimismo, Pourouma tomentosa exhibe un patrón de floración - fructificación regular y estas fases se producen entre setiembre a diciembre (Zárate et al., 2006), mientras que en la presente investigación reportamos que su patrón de floración fructificación aconteció de setiembre a marzo entre el año 2008 al 2009, de setiembre a febrero entre el 2009 al 2010 y de setiembre a enero entre el 2010 al 2011; lo que indica que esta especie puede estar en reproducción desde setiembre hasta marzo.

En cuanto a Zygia basijuga, Zárate et al. (2006), afirman que la fructificación se produce entre octubre y noviembre, y observaron también frutos en febrero y marzo, lo que indica que la fructificación duró 4 meses, lo cual coincide con este estudio.

Los meses de abril, mayo y junio son un período de sequedad del fruto y puede extenderse hasta agosto o septiembre. En este último mes, se observaron botones florales antes que se desprendieran todos los frutos secos que quedaron en el caule. Muchos individuos no hacen floración en este período inclusive hasta julio.

En cuanto a Eschweilera coriacea no se observó ni registró floración y fructificación en el período de inicio de lluvias del año 2009 y comienzos del 2010; de igual modo, a fines del año 2011, dando la impresión que tendría un periodo de no "fertilidad" en dichos períodos. Sin embargo, según Reynel et al. (2003), la floración se produce a fines de la estación de menor precipitación, entre agosto y octubre y la fructificación durante la estación de lluvias entre enero y marzo.

\section{Correlación entre las fenofases y el clima}

La floración de las especies de este estudio presentaron una baja correlación con la precipitación, estos resultados son similares a lo reportado por Zárate et al. (2006) para angiospermas leñosas de los bosques sobre arena blanca y arcilla de la Reserva Nacional Allpahuayo Mishana 
(Amazonía peruana); también por Henderson et al. (2000), para 27 especies de palmeras en la Amazonía brasilera, igualmente Khorsand et al. (2013) menciona que en Mauritia flexuosa la floración no está correlacionada a la precipitación actual, pero si lo está con la precipitación de 3 meses anteriores. Estas afirmaciones son reafirmadas por el reporte de Bentos et al. (2008) quienes no encontraron correlación entre la precipitación y la floración en la mayoría de especies pioneras cerca de Manaus (Amazonía barsilera).

La fructificación de las especies estudiadas tiene una baja correlación con la precipitación (la máxima correlación fue de 0.277 , en Iryanthera paraensis). Estos resultados son concordantes con los reportes de la mayoría de los autores (Alencar et al., 1979; Alencar, 1994; Zárate et al., 2006; y Bentos et al., 2008); a excepción de Khorsand et al. (2013), quienes reportaron para Mauritia flexuosa una correlación significativa con la precipitación actual.

Podemos concluir parcialmente que la floración y fructificación no están fuertemente influenciandas por la precipitación y temperatura media mensual en la mayoría de las especies de angiospermas en la Amazonía.

\section{AGRADECIMIENTOS}

A la Facultad de Ciencias Forestales (UNAP) por permitirnos ejecutar esta investigación en el área protegida del Campus del Centro de Investigación y Enseñanza Forestal (CIEFOR). Al Herbario Amazonense (AMAZ), por el acceso a sus instalaciones y facilidades en la identificación de las especies. Al Servicio Nacional de Meteorología e Hidrología del Perú (SENAMHI), por la información climática proporcionada. A Juan Baluarte, por la información proporcionada. A Juan J. Palacios Vega y George Pepe Gallardo Gonzales, por su ayuda en el tratamiento de la información espacial de la presente publicación. A Gabriela Vásquez La Torres, por su ayuda en la traducción de la sección en inglés. A Cristina López Wong, por su gran ayuda en la revisión del presente manuscrito.

\section{BIBLIOGRAFÍACITADA}

Alencar J. 1994. Fenología de cinco especies arbóreas tropicales de Sapotaceae correlacionada a variáveis climáticas na Reserva Ducke, Manaus. Manaus-Brazil. Acta Amazonica, 24 (3-4): 161-182.

Alencar J.; Almeida, R.; Fernández, N. 1979. Fenologia de especies florestais em floresta tropical úmida de terra firme na Amazonía central. INPA. Manaus-Brasil. Acta Amazonica, 9(1): 163-198.
Aróstegui A. \& Díaz. M. 1992. Propagación de especies forestales nativas promisorias en Jenaro Herrera. Instituto de Investigación de la Amazonia Peruana-IIAP. Iquitos, Perú. 119pp.

Baluarte J. 1996. Comportamiento fenológico preliminar de cuatro especies forestales de áreas inundables. Folia Amazónica, 7 (1-2): 205-217.

Bentos, T.; Mesquita, R.; Williamson, G. 2008. Reproductive Phenology of Central Amazon Pioneer Trees. Tropical Conservation Science, 1 (3):186-203.

Bullock, S.; Beach, J.; Bawa, K. 1983. Episodic flowering and sexual dimorohism in Guarea rhopalocarpa in a Costa Rican rain forest. Ecology, 64(4): 851-861.

Castro, R. 1987. Fenologia de dez Espécies Arbóreas Nativas do Cerrado de Brasilia-DF. Brasil Florestal, 62 Out./Nov./Dez: 23-29.

Flores, M. 2008. Fenología de especies vegetales amazónicas a partir del conocimiento registrado en los herbarios. Tesis Doctoral. Universidad Nacional de Trujillo. Trujillo, Perú. 76pp.

Font, P. 1985. Diccionario de Botánica. Ed. LABOR, S. A. Barcelona-Madrid. 1244pp.

Foster, R. 1990. Ciclo Estacional de Caída de Frutos en la Isla de Barro Colorado. In: Rand, A.; Windsor D.; Leigh, J.; Giles, E. (Eds.) Ecología de un bosque tropical: ciclos estacionales y cambios a largo plazo. p. 219-242.

Freitas, L. \& Peter, L. 2000. Fenología de Unonopsis floribunda Diels y Oxandra sphaerocarpa R.E. Fries (Annonaceae) en bosques de la planicie inundable de Jenaro Herrera, Loreto, Perú. Folia Amazónica, 10 (1-2):183-200.

Gautier, L. \& Spichiger, R. 1986. Ritmos de reproducción en el estrato arbóreo del Arbortum Jenaro Herrera (Provincia de Requena, Departamento de Loreto, Perú) Contribución al estudio de la flora y de la vegetación de la Amazonia Peruana. Candollea, 41: 193-207.

Henderson, A.; Fischer, B.; Scariot, A.; Whitaker, M.; Pardini, R. 2000. Flowering phenology of a palm community in a central Amazon forest. Brittonia, 52 (2): 149-159.

Khorsand, R.; Imbrozio, R.; Koptur, S. 2013. How do habitat and climate variation affect phenology of the Amazonian palm, Mauritia flexuosa?. Journal of Tropical Ecology, 29:255-259.

López, R. 1981. Estudio Silvicultural del tornillo (Cedrelinga cateniformis Ducke). Revista Forestal del Perú, 10 (1-2): 1-7.

Pérez, F. \& Martinez-Laborde, J. (1994). Introducción a la Fisiología Vegetal. Ediciones Mundi-Prensa, Madrid. 123pp. 
Pfrommer, A. \& Würzburg, F. 2009. Seed dispersal ecology of Leonia cymosa (Violaceae) in the rain forest of Eastern Ecuador. Dissertation zur Erlangung des aturwissenschaftlichen Doktorgrades der Bayerischen JuliusMaximilians-Universität Würzburg vorgelegt. $143 p p$.

Rathcke, B. \& Lacey, E. 1985. Phenological Patterns of terrestrial plants. Annual Reviews Ecol. Syst., 16: 179-214.

Reynel, C.; Pennington, T.; Pennington, R.; Flores, C.; Daza, A. 2003. Árboles útiles de la Amazonia peruana. Un Manual con apuntes de identificación, ecología y propagación de especies. 509pp.

Ruiz, F. 2000. "Evaluación fenológica de diez especies forestales de los bosques productivos de la Comunidad Nativa Santa Mercedes - Rio Putumayo; Loreto-Perú". Tesis para optar el título profesional de Ingeniero Forestal. UNAP. Iquitos-Perú. 128pp.

Rutter, R. 1990. Catálogo de plantas útiles de la Amazonia Peruana. Ministerio de Educación. Instituto Lingüístico de Verano. Lima, Perú. 349pp.

Torres, G. 2001. Avances sobre la Fenología de 18 especies Forestales en el Bosque de la Llanura Aluvial Inundable de Jenaro Herrera. Informe Técnico. Instituto de Investigaciones de la Amazonía Peruana. Iquitos, Perú 53pp.
Tello, R. 1996. Fenología de cinco especies da familia Myristicaceae na Reserva Ducke, Manaus-Amazonas. Dissertação de Mestrado. INPA/UFAM. Manaus, Brasil. 110pp.

Vásquez, M. 1997. Flórula de las Reservas Biológicas de Iquitos, Perú. Missouri Botanical Garden Press. St. Louis, USA.1046pp.

Vásquez, M. 1989. Plantas útiles de la Amazonía Peruana. Iquitos, Perú. 195pp.

Vásquez, R. \& Rojas, R. 2006. Plantas de la Amazonía Peruana. Clave de Identificación de Gymnospermae y Angiospermae. Arnaldoa, 13 (1): 1-258.

Villasana, R. \& A. Suárez. 1997. Estudio fenológico de dieciséis especies forestales presentes en la Reserva Forestal Imataca Estado BolívarVenezuela. Revista Forestal Venezolana, 41 (1): 13-21.

Zárate, R.; Amasifuen, C.; Flores, M. 2006. Floración y fructificación de plantas leñosas en bosques de arena blanca y suelo arcillosos en la Amazonía Peruana. Revista Peruana de Biología, 13 (1): 95-102.

Recibido: 10 de julio del 2015

Aceptado para publicación: 15 de agosto del 2015 
